\title{
Concept identification strategies used for positive and negative instances'
}

\author{
J. PETER DENNY and JOHN G. BENJAFIELD, ${ }^{2}$ University \\ of Western Ontario, London, Canada
}

Previous investigators have speculated about strategies of reasoning which might account for poorer proficiency in concept identification from negative than from positive instances. In this study novel dependent variables were used to provide a broad data base from which Ss' strategies could be inferred. These measures were Ss' responses indicating their conclusions about the concept as they developed during the course of the task, and Ss' reports, using stimulated recall after the task, of their reasoning used to infer these conclusions from the information given in the task. Three types of strategies were discovered: formal strategies consisting of correct deductive reasoning, concrete strategies consisting of unintegrated inferences from particular pieces of information, and nonprocessing strategies in which no inferences were drawn. Ss receiving a negative instance task used correct formal strategies less frequently and incorrect concrete strategies more frequently than Ss receiving a positive instance task. They did so because of a strong tendency to infer that an attribute absent from an instance was not in the concept.

Concept identification tasks present two major kinds of information to Ss, that specifying the attributes and values in the instances, and that specifying whether the instances are or are not examples of the concept. Studies of the latter kind of information have shown that $S s$ form concepts less proficiently from nonexamples (negative instances) than they do from examples (positive instances) (e.g., Hovland \& Weiss, 1953). Various investigators have attempted to explain this finding by suggesting strategies of concept identification which Ss might have used (e.g., Fryatt \& Tulving, 1963). However, these suggestions were speculative because the studies did not employ dependent variables from which strategies could be inferred. Those which were used were measures of gross proficiency in identifying the concept. The present study used novel dependent variables especially developed to assess strategies, so as to provide an adequate empirical basis to account in terms of strategies for the difference in proficiency between positive and negative instances.

\section{SUBJECTS AND PROCEDURE}

Subjects from introductory psychology classes were randomly assigned to either a task containing positive instances or one containing negative instances so that there were $15 \mathrm{Ss}$ in each group. The tasks, shown in Table 1 , utilized eight attributes which were the letters, A through $H$. The letters were either present or absent in an instance, which was a row, e.g., ABC EFGH. The conjunctive concept to be identified contained four attributes, A, D, E, and $G$.

The positive task contained five positive instances, each marked + , of which the first was a focus instance containing all the attributes, and the others had one different attribute absent from among those not in the concept. The negative task contained the same first positive instance as the positive task, followed by four negative instances, each marked -, and having one different attribute absent from among those in the concept. The amounts of information in each instance and in each task were equal, since each instance after the first one established the relation of one attribute to the concept.

The instructions explained attributes, conjunctive concepts, positive and negative instances, but did not say how many attributes were in the concept. The task was presented to $S$ one instance at a time. Previously presented instances remained in view in order of presentation.

\section{ASSESSMENT OF STRATEGIES}

The strategies of reasoning which Ss used to transform the information given in the task into conclusions about the concept were assessed by means of two basic dependent variables, especially developed for the investigation of strategies. Although they have been previously used for other purposes (Denny, 1966; Denny \& Benjafield, 1968) this is the first report of their primary use.

The first measure was designed to enable $S$ to keep a record of his conclusions about the concept as he gradually inferred them from the successive pieces of information provided in the task, in such a way that the responding involved would not interfere with the thinking process. Ss were required to state for each attribute either that they concluded it was in the concept (symbolized I), or not in the concept (symbolized $\mathrm{N}$ ). or that they did not know whether or not it was in the concept (symbolized ?). They drew these conclusions for every attribute after the presentation of every instance, and recorded them on a record sheet using the symbols $I, N$, and ?. The second measure, which was based upon the first, was S's report of his reasoning used to infer each of his recorded conclusions given at the end of the task. S's report was based upon stimulated recall since he was exposed to the complete task and the complete record of his $I, N$, and ? responses.

Subjects' strategies were inferred by objectively describable procedures primarily from Ss' postexperimental interview statements, and secondarily from their $\mathrm{I}, \mathrm{N}$, and ? responses. Preliminary examination of the interviews revealed important facts about Ss' strategies. Strategies were seen to be composed of components, each of which had a premise and conclusion structure, e.g., premise: "if an attribute is absent from a positive instance," conclusion: "then it is not in the concept." The premises referred to the information used by $S$, and the conclusions referred to the inferences drawn from that information.

Subjects' interviews were examined to discover the variety of premises and conclusions therein. Approximately 150 different statements, some of premises and some of conclusions, were discovered. These were expressed in standard terms using a standard grammar, as illustrated in the example above. Then, the individual S's statements of premises and conclusions were translated into standard statements. This translation was completely and objectively described in a set of conversion tables giving all of S's statements which were set equivalent to each standard statement. Work is under way to replace these conversion tables with scoring programs which can convert new statements from subsequent studies.

Subjects' interviews as translated into standard statements were then examined to discover what patterns of statements constituted the strategies of concept identification. A number of strategies were discovered, a complete report of which will be forthcoming; however, for the present paper these were grouped into three types described in the results. The types were objectively described in terms of the various standard statements (both premises and conclusions) which comprised them, and individual S's were scored for the type of strategy they used. Inferences about strategy types based upon interview statements were verified by noting that 29 out of 30 Ss showed the pattern of $\mathrm{I}, \mathrm{N}$, and ? responses, made during the course of the task, which would be expected for their strategy type. 
ASSESSMENT OF

CONCEPT IDENTIFICATION PROFICIENCY

Subjects' proficiency was measured by the number of attributes which he correctly included in or excluded from the concept when asked for his conclusions at the end of the task. Since there were eight attributes, scores could range from 0 to 8 .

\section{RESULTS}

As previously reported (Denny \& Benjafield, 1968), Ss receiving the positive task drew significantly more correct conclusions that attributes were included in or excluded from the concept, $\bar{X}=7.3$, than did Ss receiving the negative task, $\bar{X}=2.7, U=33, n_{1}=n_{2}=15, p<.002$.

The most important discovery revealed in the data was that Ss' strategies fell into three broad types, two of which appeared equivalent to Piaget's formal and concrete levels of cognitive functioning (Flavell, 1963). The formal type consisted of those strategies in which Ss processed the information given in the task in a fully integrated manner by means of correct deductive reasoning. The concrete type consisted of those strategies in which Ss drew particular inferences whether correct or incorrect, from particular pieces of information in the task, but did not thoroughly integrate the information or their inferences from it. The third type were nonprocessing strategies in which Ss responded to the information in the task but did not process it, i.e., they drew no inferences from it.

Table 1

The Concept Identification Tasks

\begin{tabular}{|c|c|}
\hline Positive Task & Negative Task \\
\hline 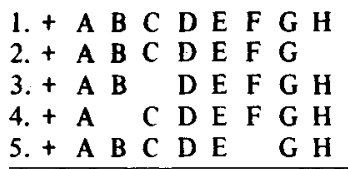 & $\begin{array}{lllllllll}\text { 1. + } & \text { A } & \text { B } & \text { C } & \text { D } & \text { E } & \text { F } & \text { G } & \text { H } \\
2 .- & \text { A } & \text { B } & \text { C } & \text { D } & & \text { F } & G & H \\
3 .- & \text { B C } & \text { D E } & \text { F } & \text { G } & \text { H } \\
\text { 4. - } & \text { A } & \text { B } & \text { C } & & \text { E } & \text { F } & \text { G } & \text { } \\
5 .- & \text { A } & \text { B } & \text { C } & \text { D E E } & \text { F } & & \text { H }\end{array}$ \\
\hline
\end{tabular}

Examination of the frequency of Ss using these three types of cognitive functioning, as shown in Table 2, revealed that 13 of the 15 negative-task Ss used the concrete or nonprocessing types whereas only 8 of the 15 positive-task Ss did so, a result not quite significant at the .05 level by the Fisher exact probability test. Data from two additional groups of $15 \mathrm{Ss}$ each were examined, and the same effect was found, $p<.05$ by the Fisher exact probability test. Thus, Ss receiving negative instances used lower levels of cognitive functioning more frequently than $S s$ receiving positive instances.

Table 2 also shows the median concept identification proficiency score associated with each strategy class, i.e., the median number of attributes correctly included in or excluded from the concept at the end of the task. It can be seen that formal strategies were always associated with proficient concept identification, as would be expected since only logically correct strategies qualify for this type. However, for the concrete type, strategies involving both correct and incorrect inferences may occur. Examination of the data for

Table 2

Number of Ss and Median Number of Attributes Correctly Included in and Excluded from the Concept for Three Strategy Types

\begin{tabular}{|c|c|c|c|c|c|c|c|}
\hline \multicolumn{2}{|c|}{ Strategy } & \multicolumn{3}{|c|}{ Positive task } & \multirow[b]{2}{*}{ f } & \multicolumn{2}{|c|}{ Negative task } \\
\hline Type & Inference & f & Median & Range & & Median & Range \\
\hline Formal & & 7 & 8 & $6-8$ & 2 & 7 & $6-8$ \\
\hline \multirow[t]{3}{*}{ Concrete } & Correct & 7 & 8 & $4-8$ & 2 & 8 & none \\
\hline & Incorrect & 0 & - & - & 7 & 0 & $0-1$ \\
\hline & from lst & 1 & 4 & none & 2 & 4 & none \\
\hline \multicolumn{2}{|c|}{ Nonprocessing } & 0 & - & - & 2 & 1 & $0-2$ \\
\hline
\end{tabular}

this type revealed that only 2 out of 11 negative-task Ss used concrete strategies which were associated with perfect median proficiency scores of 8 , whereas 7 out of 8 positive-task Ss did so, $p<.01$ using the Fisher exact probability test. Thus, negative-task Ss more frequently used incorrect concrete strategies which did not yield the correct concept. Proficiency data for Ss using nonprocessing strategies were regarded as meaningless since these Ss did not attempt to identify the concept.

\section{DISCUSSION}

The results show that Ss handle negative instances less proficiently for two reasons. First, Ss given negative instances used concrete and nonprocessing strategies more frequently and consequently formal strategies less frequently than Ss given positive instances. Since formal strategies make optimal use of the information given, less frequent use of them accounts for lowered proficiency. Second, Ss given negative instances employ incorrect concrete strategies more frequently than do those given positive instances. We may summarize by saying that negative instances led to less use of correct formal strategies and more use of incorrect concrete strategies.

This difference between positive and negative instances arises from the preferred inference which $S$ s make from the most important piece of information in the task, the absence of an attribute from each instance. Leaving aside the 3 Ss who do not use this information but use the first instance instead, of the 27 remaining Ss in both groups, 23 make the inference that an absent attribute is not in the concept. This inference is a correct one when applied to positive instances but an incorrect one when applied to negative instances. Of the $27 \mathrm{Ss}$, all 14 of those who received the positive-instance task used this inference, as part of either a formal or a concrete strategy, and all formed the concept proficiently. However, 9 of the 13 Ss receiving the negative task also used this inference, in all cases as part of a concrete strategy, and all of them obtained low proficiency scores. The remaining 4 negative-task Ss used the correct inference for negative instances, an absent attribute is in the concept, in two cases as part of a concrete strategy and in two cases as part of a formal strategy, and all of them formed the concept proficiently. These results are consonant with those of Wickelgren \& Cohen (1962) who, although they did not describe strategies, did find that Ss drew more correct inferences from information, analogous to the information in the present task that an attribute was absent, when it occurred in positive rather than in negative instances. From the above discussion we see that $S$ s receiving negative instances used fewer correct formal strategies and more incorrect concrete strategies because they used a strongly preferred inference which was incorrect for negative instances. Thus, differences in proficiency for positive and negative instances depend largely upon Ss using the inference concerning absent attributes that is appropriate for the type of instance encountered. Denny \& Benjafield (1968) have suggested that use of a correct or incorrect inference is related to making a correct or incorrect interpretation of the information that an instance is positive or negative.

The results of this study are quite different from the speculations about strategies offered by previous authors to explain the difference in proficiency for positive and negative instances. Glanzer, Huttenlocher, \& Clark (1963) suggested that negative and positive instances, which are preceded by a positive focus instance as in the present study, impose a different memory load upon Ss. This explanation is excluded here because Ss did not have to remember either the information in the task or their previous conclusions. Fryatt \& Tulving (1963) claimed that their Ss were using inductive reasoning as a strategy for positive instances, and speculated that Ss receiving negative instances tried to convert the information therein into information to which the positive 


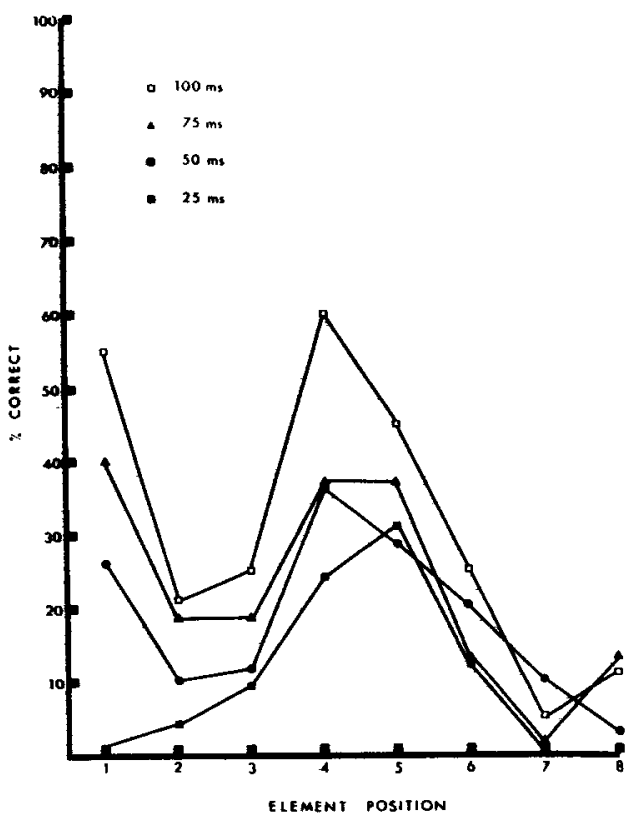

Fig. 2. Interaction of Exposure by Element Position for localization scores in the symmetrical condition.

The critical interaction of Exposure by Position for localization scores in the symmetrical condition is shown in Fig. 2. The data indicate that as exposure increases, elements in the left periphery of the visual field are recalled more readily. What is happening here, it seems, is that increasing exposure allows the left-right scan to start further at the left. The percentages of elements correctly localized in the symmetrical condition from Position 1 were: $1(25 \mathrm{msec})$, $26(50 \mathrm{msec}), 40(75 \mathrm{msec})$, and $55(100 \mathrm{msec})$. The mean left-right order of report (Bryden, 1966) also increased over exposure: $.71(25 \mathrm{msec}), .74(50 \mathrm{msec}), .79(75 \mathrm{msec})$, and $.86(100 \mathrm{msec})$. The absence of this interaction in the analysis by identification scores can be attributed to a high degree of identification of elements but in incorrect element positions, thus cancelling any position effects.

The interaction of Exposure by Material for identification scores and the absence of same for localization scores would also indicate digits are better identified and recalled (irrespective of position) than letters, but scoring by localization reduces this discrepancy. Any response-bias and guessing, probably inherent in identification scores and expectedly greater for digits (9) than for letters (13), would also be reduced by localization criteria.

Generally, the data parallel those of Crovitz \& Schiffman (1965), Bryden (1966), and Mathewson et al (1968). The experimental conditions employed here may account for the high accuracy of the middle (4 and 5) positions relative to the extreme left position (1) not found by the authors cited. The likelihood of Ss having a set to scan from far-left to far-right throughout experimental trials, possible in the conditions employed by these authors, is reduced by randomizing asymmetrical with symmetrical presentations within trialblocks. This may also account for the clear Exposure by Position interaction for localization scores, suggested by their data (the $F$ of 2.96 with $\mathrm{df}=7 / 133$ given by Mathewson et al, would appear to be highly significant in this respect, though they state it is not significant).

\section{REFERENCES}

BRYDEN, M. P. Accuracy and order of report in tachistoscopic recognition. Canadian Journal of Psychology, 1966, 20, 262-272.

CROVITZ, H. F., \& SCHIFFMAN, H. R. Visual field and the letter span. Joumal of Experimental Psychology, 1965, 70, 218-223.

CROVITZ, H. F., SCHIFFMAN, H., LIPSCOMB, D. B., POSNICK, G., REES, J., SCHAUB, R., \& TRIPP, R. Identification and localization in the letter span. Canadian Journal of Psychology, 1966, 20, 455-461.

MATHEWSON, J. W., MILLER, J. C., JR., \& CROVITZ, H. F. The letter span in space and time. Psychonomic Science, 1968, 11, 69-70.

SPERLING, G. The information available in brief visual presentations. Psychological Monographs: General \& Applied, 1960, 74, (11, Whole No. 498),

(Continued from page 278)

inductive strategy could be applied. No evidence for such conversion was found in the present study. Huttenlocher (1962) suggested that Ss might use formal strategies which required more steps of reasoning for certain negative instance problems than for certain positive instance problems. Although this suggestion cannot be directly tested by the present data, since the present tasks are most similar to ones for which Huttenlocher hypothesizes an equal number of reasoning steps, the finding that lowered proficiency is only associated with concrete strategies does not support Huttenlocher's notion that lowered proficiency can occur for formal strategies involving increased numbers of reasoning steps.

\section{REFERENCES}

BRUNER, J. S., GOODNOW, J. J., \& AUSTIN, G. A. A study of thinking. New York: Wiley, 1956.

DENNY, J. P. Effects of anxiety and intelligence on concept formation. Journal of Experimental Psychology, 1966, 72, 596-602.

DENNY, J. P., \& BENJAFIELD, J. G. Interpretation of information that an instance is positive or negative in concept identification. Psychonomic Science, 1968, 12, 283-284.
FLAVELL, J. H. The developmental psychology of Jean Piaget. New York: Van Nostrand, 1963.

FRYATT, M. J., \& TULVING, E. Interproblem transfer in identification of concepts involving positive and negative instances. Canadian Journal of Psychology, 1963, 17, 106-117.

GLANZER, M., HUTTENLOCHER, J., \& CLARK, W. H. Systematic operations in solving concept problems. Psychological Monographs, 1963,77 (Whole No. 564).

HOVLAND, C. I., \& WEISS, W. Transmission of information concerning concepts through positive and negative instances. Journal of Experimental Psychology, 1953, 45, 175-182.

HUTTENLOCHER, J. Some effects of negative instances on the formation of simple concepts. Psychological Reports, 1962, 11, 35-42.

WICKELGREN, W. A., \& COHEN, D. H. An artificial language and memory approach to concept attainment. Psychological Reports, $1962,10,815-827$.

\section{NOTES}

1. Based on data collected for an M.A. thesis by the junior author, supervised by the senior author, and submitted to the Faculty of Graduate Studies, University of Western Ontario. This research was partially supported by National Research Council of Canada Grants APA-81 and APA-137.

2. Now at Rutgers: The State University, Newark, N. J. 